\title{
QED vs. classical radiation reaction in the transition regime
}

M. Vranic', T. Grismayer, J. L. Martins, R. A. Fonseca, and L. O. Silva

Citation: 1777, 050006 (2016); doi: 10.1063/1.4965629

View online: http://dx.doi.org/10.1063/1.4965629

View Table of Contents: http://aip.scitation.org/toc/apc/1777/1

Published by the American Institute of Physics 


\title{
QED vs. Classical Radiation Reaction in the Transition Regime
}

\author{
M. Vranic ${ }^{1, \text { a) }}$, T. Grismayer ${ }^{1}$, J. L. Martins ${ }^{1}$, R. A. Fonseca ${ }^{1,2}$ and L.O. Silva ${ }^{1}$ \\ ${ }^{1}$ GoLP/Instituto de Plasmas e Fusão Nuclear, Instituto Superior Técnico, Universidade de Lisboa, Lisbon, \\ Portugal \\ ${ }^{2}$ DCTI/ISCTE, Insituto Universitário de Lisboa, 1649-026 Lisbon, Portugal \\ a) Corresponding author: marija.vranic@ist.utl.pt
}

\begin{abstract}
We focus our analysis in the properties of an electron beam during/after collision with an intense pulse. The additional energy spread introduced by the stochastic nature of QED emission can be balanced by the average energy loss leading to overall energy spread reduction even in the QED regime.
\end{abstract}

\section{INTRODUCTION}

Multi-petawatt laser systems presently available can provide intensities of $10^{22} \mathrm{~W} / \mathrm{cm}^{2}$, at which the physics can no longer be assumed purely classical and appropriate quantum corrections must be taken into account. Near-future facilities [1] are expected to provide even higher intensities (on the order of $10^{24} \mathrm{~W} / \mathrm{cm}^{2}$ ) and there has been a significant research effort towards understanding the laser-plasma interaction in such conditions [28]. A powerful tool that supports theoretical studies of laser-matter interaction and helps the design of experiments are particle-in-cell (PIC) codes, which self-consistently use Maxwell's equations to advance the electromagnetic fields and the Lorentz force to move the charged particles. However, the standard PIC algorithm is entirely classical and does not incorporate any quantum effects. In order to expand the validity of our PIC code OSIRIS [9] for studies at higher intensities, we have included two additional modules: a classical radiation reaction module based on the Landau \& Lifshitz [10] equation, and a quantum electrodynamics (QED) module that has discrete photon emission (non-linear Compton scattering) and Breit-Wheeler electron-positron pair production [11]. In this manuscript, we study the differences between the two pictures in the transition from the classical to the quantum-dominated regime, with parameters of interest for near-future experiments. Our configuration considers relativistic electron beams $\left(\mathrm{GeV}\right.$-level) and ultra-intense lasers $\left(10^{21}-10^{22} \mathrm{~W} / \mathrm{cm}^{2}\right)$ in a head-on collision. The study compares the evolution of the main electron beam properties (energy loss, energy spread, divergence) from the QED and classical perspectives. Lasers of various durations are considered to identify the role of the interaction length.

\section{RADIATION REACTION FOR AN ELECTRON IN AN ELECTROMAGNETIC WAVE}

In our previous work [12], we have studied the classical radiation reaction of a particle that emits continuous radiation and looses energy as a consequence of the emission. Therefore, the energy of the particle is a smooth function of time. This approximation is considered valid as long as the energy of a single emitted photon is small compared with the energy of the particle. In these conditions the highly energetic electrons radiate more than the electrons with lower energy and therefore the energy spread of an electron beam is reduced during the laser interaction.

In QED, radiation is a discrete stochastic process and this impacts the particle trajectory in a distinct manner from the continuous emission. The probabilities of the various processes in an electromagnetic plane wave are based on Volkov [13] states where the quantum-transition probability is evaluated taking into account the interaction between the particle and the background wave. In the event of emission, the electron loses some energy to the photon; otherwise, the electron momentum and energy remain unaltered. If every single photon

Advanced Accelerator Concepts 2014

AIP Conf. Proc. 1777, 050006-1-050006-5; doi: 10.1063/1.4965629

Published by AIP Publishing. 978-0-7354-1439-6/\$30.00 
radiated has a small energy compared with the electron energy, and there is a large number of radiated photons, we obtain the classical limit. The main difference between the two descriptions is that the QED approach, in principle, accounts for radiated high-energy photons even in a setup where the cross-section for Compton scattering is small (i.e. the average energy loss of the particle is negligible). Hence, the stochastic nature of emission leads to a diffusion around the mean value in the electron distribution function, as it was reported in refs. [7, 8]. This is opposite of what is predicted in a classical radiation reaction scenario.

The total probability of a process by a single particle is relativistically invariant and depends on the normalised vector potential $a_{0}=e E /\left(m c \omega_{0}\right)$ and an invariant parameter $\chi\left(\chi_{e}\right.$ for electrons and $\chi_{\gamma}$ for photons) defined by:

$$
\chi_{e}=\frac{\sqrt{p_{\mu} F^{\mu \nu}}}{m c E_{s}}, \chi_{\gamma}=\frac{\sqrt{k_{\mu} F^{\mu \nu}}}{m c E_{s}},
$$

where $p_{\mu}$ is the particle 4-momentum, $k_{\mu}$ is the photon wave 4-vector, $F^{\mu v}$ the electromagnetic tensor, $E_{s}=m^{2} c^{3} /(e \hbar)$ the Schwinger critical field, $m$ and $e$ are the electron mass and charge, $c$ is the speed of light and $\omega_{0}$ is the angular frequency of the wave. The differential probability rate of photon emission by nonlinear Compton scattering is then given [14] by

$$
\frac{d^{2} P}{d t d \chi_{\gamma}}=\frac{\alpha m c^{2}}{\sqrt{3} \pi \hbar \gamma \chi_{e}}\left[\left(1-\xi+\frac{1}{1-\xi}\right) K_{2 / 3}\left(\chi^{*}\right)-\int_{\chi^{*}}^{\infty} d x K_{1 / 3}(x)\right],
$$

where $\chi^{*}=2 \xi /\left(3 \chi_{\mathrm{e}}(1-\xi)\right)$ and $\xi=\chi_{\nu} / \chi_{\mathrm{e}}$. For $\xi<<1$ the total radiated power reduces to the classical synchrotron.

The OSIRIS QED module introduces the previous differential emission probability rate in the OSIRIS framework. At particle push-time, the probability of radiating a photon is evaluated, and if the event occurs, the radiated photon energy is selected to obey the distribution given by Eq. (2). The photon is assumed to be radiated in the direction of the particle motion. There is no radiation reaction on particles if no photons are emitted, but if the emission occurs, the particle energy and momentum are updated to account for the energy lost to the emitted photon. The energy is conserved in the loop.

\section{SIMULATION SETUP}

Here we investigate the evolution of energy spread and divergence of an initially almost ideal electron bunch that interacts with different laser pulses. This allows for following exactly the divergence and energy spread increase and decrease in time, as well as their dependence on different laser lengths and shapes. We choose two electron bunches with mean energies of $0.5 \mathrm{GeV}$ and $0.85 \mathrm{GeV}$ and a small thermal momentum spread (thermal velocity $\gamma v_{t h}=0.2 \mathrm{c}$ ) equal in all directions that provides a very narrow divergence and energy spread. Both beams have the same lengths of $25.0 \mathrm{c} / \omega_{0}$ (which corresponds to $13.3 \mathrm{fs}$ ). These bunches collide head-on with seven different lasers. All lasers are transversally plane waves with longitudinal envelopes that have the same rise and fall functions, but their duration can be varied by introducing a flat part in the middle. Therefore, $\tau_{\text {rise }}=$ $\tau_{\text {fall }}=50.0 \omega_{0}^{-1}$ (which corresponds to $26.6 \mathrm{fs}$ ) and the flat part $\tau_{\text {flat }}$ varies between $0.0 \omega_{0}^{-1}$ and $300.0 \omega_{0}^{-1}$ with a step of $50.0 \omega_{0}^{-1}$ (seven different total pulse durations $\tau=\tau_{\text {flat }}+\left(\tau_{\text {rise }}+\tau_{\text {fall }}\right) / 2$ ). The normalising frequency $\omega_{0}=1.88 \times 10^{15} \mathrm{rad} / \mathrm{s}$ corresponds to a laser with wavelength $\lambda=1 \mu \mathrm{m}$. Here, the interaction between the particles of the beam is negligible and since the laser field does not have $x_{2}$ and $x_{3}$ spatial dependence, all the particles in the simulation are subject to the same conditions. The simulations are performed in two spatial dimensions (2D) with a box size of $500.0 \times 20.0 \mathrm{c} / \omega_{0}$, and $5000 \times 200$ simulation cells. The boundary conditions were periodic in the $x_{2}$ direction (perpendicular to the laser propagation direction $x_{1}$ ) and open in the $x_{1}$ direction. The simulation timestep was $d t=0.04 \omega_{0}^{-1}$ with sixteen particles per cell. Having many particles in the beam $(\simeq 1$ milion $)$ provides us with a good statistical sample to study the evolution of the energy spectra and the divergence of the electrons.

\section{EVOLUTION OF THE ELECTRON ENEGY SPECTRA}

We first consider the temporal evolution of energy spectrum of the beam as it interacts with the intense lasers. The results of our simulations are summarized in Fig. 1 which shows the electron energy spectra after the interaction. All the spectra, as expected, are wider than the initial nearly delta-function distribution. The first striking fact here is that after interaction with longer lasers, the final energy spread of the electron beam seems to be narrower than after interacting with shorter lasers. This hints that for longer interaction there is a turning point where after the initial increase in the spectral width associated with the quantum nature of the radiation 
process in the early stages of the interaction, the width reduces again as predicted for classical radiation reaction [12].

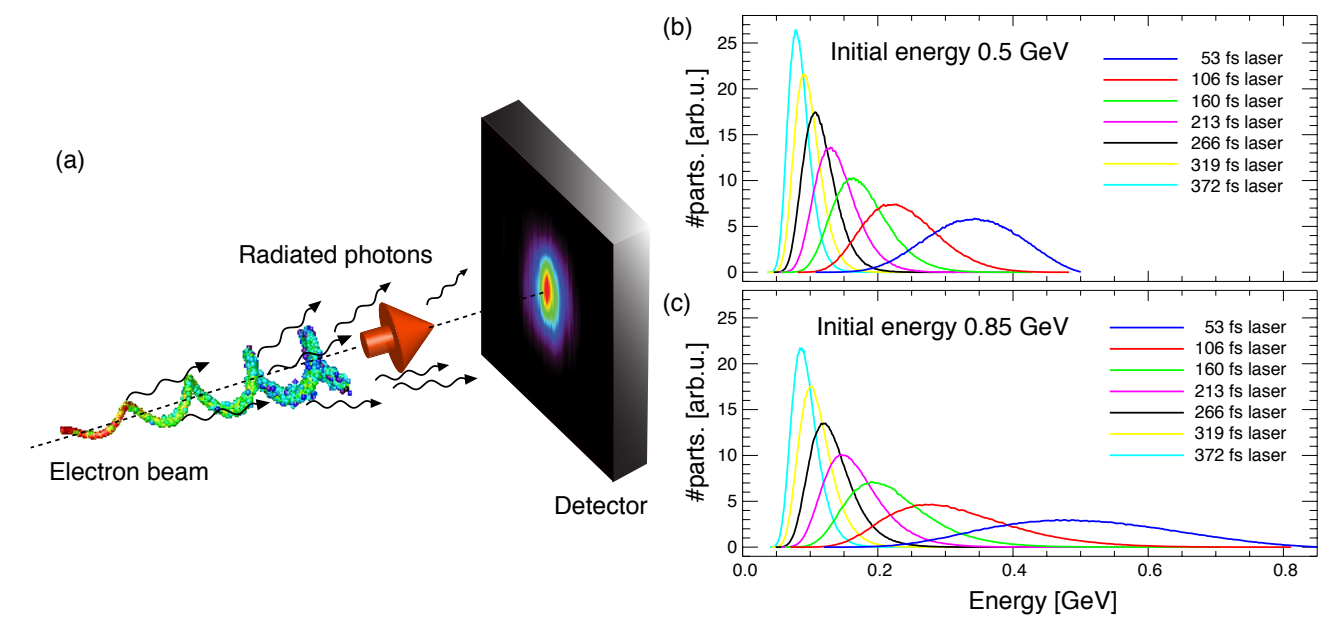

FIGURE 1. (a) Scheme (counter propagating laser not depicted) with individual events of photon emission that cause a noncontinuous energy loss; energy spectra after shutdown of the laser starting from (b) a $0.5 \mathrm{GeV}$ electron beam and (c) a 0.85 $\mathrm{GeV}$ electron beam.

Therefore, examining the temporal evolution of the energy spectra and its width could reveal what is the moment in time where the QED radiation reaction changes from widening the beam spectrum to narrowing it. We define the beam width at a time $t$ as the standard deviation in energy over all the particles

$$
\sigma(t)=\sqrt{\frac{1}{N} \sum_{i=1}^{N}\left(\langle\gamma(t)\rangle-\gamma_{i}(t)\right)^{2}},
$$

where $N$ is the total number of particles, $\left\langle\gamma(t)>\right.$ is the mean energy of the entire electron beam at the time $t, \gamma_{i}(t)$ is the energy of a single particle at the same time, and $i$ counts all the particles. The analysis of the beam spectral width evolution through the interaction time confirms the previous assumption: the first effect of the interaction is to broaden the spectrum to a certain value. If the laser is short enough, the spectrum stays broad. However, if the laser used is longer, then there is a specific point in time where the spread starts decreasing. If the electron beam energy can be approximated by a Gaussian distribution [7] for a laser wavelength of $1 \mu \mathrm{m}$ this happens when (the derivation will be presented elsewhere):

$$
\sigma(t)^{2}[M e V]=3.6 \times 10^{-6} a_{0}\langle\gamma(t)\rangle^{3}[M e V]
$$

Let us comment on the underlying physics involved. The emission probability is initially almost the same for all the particles, because they have similar momenta and they experience the same field, and therefore all have the same $\chi_{e}$ parameter. However, since the emission process is probabilistic, there could be still some particles that do not emit any photon at this point. Also, the emitting particles do not emit at the same photon energy there is a wide spectrum of photons with different differential probabilities of emission. The electrons that radiate higher energy photons lose immediately a bigger fraction of their energy compared with the electrons that radiate smaller energy photons. Therefore, the widening of the electron spectra is natural to expect at early times in this configuration. Later, the electron spectrum is already wide, but the laser is still interacting with the beam. Further emission can take place, but the probability of emission is no longer equal for all the particles the particles that have a higher $\chi_{e}$ have a higher probability to emit a high-energy photon compared to the electrons with lower energy (and therefore lower $\chi_{e}$ ). The lower energy electrons still can emit, but their photons are much more likely to be in the lower energy part of the spectrum. This is what then leads to the shrinking of the electron beam energy spectrum.

\section{ELECTRON BEAM DIVERGENCE}

In addition to the electron energy, we can also evaluate the impact of the laser interaction on the electron beam divergence. We define the weighted average of the deflection angle from the main propagation direction as 


$$
\langle\tan \theta\rangle=\frac{\sum_{i=1}^{N} q_{i}\left(\frac{p_{\|}}{p_{\perp}}\right)_{i}}{\sum_{i=1}^{N} q_{i}},
$$

where $N$ is the total number of simulation particles, $q_{i}$ is the charge weight of the $i$-th particle, and $\left(p_{\perp} / p_{\| I}\right)_{i}$ is the ratio of the transversal to the longitudinal momentum with respect to the direction of laser propagation. For small angles, $\tan \theta \simeq \theta$, and the average divergence shown in Fig. 2 is determined with this approximation.

Figure 2 shows the evolution of the electron bunch divergence over time. We can see that for all cases there is a smooth rise that corresponds to the laser envelope rise, then there is a linear-rise stage where the laser amplitude is constant, and then the smooth fall that corresponds to the laser envelope fall. The first and the last stages happen also in a purely classical scenario where the total energy radiated by electrons is negligible - a laser introduces a perpendicular momentum on electrons as they are oscillating in the electric field of the laser pulse, but this change is temporary and when the interaction is over, the beam divergence returns to the initial state due to the conservation of the canonical momentum of the electron in the laser field. However, without a significant emission, the electron beam in the constant amplitude section of the laser envelope would not exhibit any rise in the average angle. In a semi-classical case where radiation reaction is significant, but can be described with continuous models, we do expect a linear rise. To understand why, let us consider what happens when a particle interacts with a circularly polarised plane wave. First, there is a perpendicular momentum of the particle that is equal to the wave normalised vector potential $a_{0}$, which also results from the canonical momentum conservation. We assume a case similar to one of ours, so here $a_{0}=27$ and $p_{\perp} \simeq 27$. Second, the initial particle energy is on the order of a $0.5-1.0 \mathrm{GeV}$, which means that $\gamma_{0} \gg a_{0}$ and $p_{\|}{ }^{2}=\gamma^{2}-a_{0}{ }^{2}-1 \simeq \gamma^{2}$. In this case the average angle that a single electron makes with the direction of laser propagation can be approximated by $\theta \simeq a_{0} / \gamma$. The fact that there is strong emission makes the relativistic factor $\gamma$ decrease, and the rate of this decrease in a linearly polarised wave is given [12] by $d \gamma / d t=-\alpha \gamma^{2}$, where $\alpha=4 e^{2} \omega_{0}^{2} a_{0}^{2} /\left(3 m c^{3}\right)$. For a circularly polarised wave $\alpha$ needs to be multiplied by 2 . By integrating we get $\gamma=\gamma_{0} /\left(1+2 \alpha \gamma_{0} t\right)$ in the field of a circularly polarised wave which gives us a linear function for the angle as a function of time: $\theta \simeq\left(a_{0} / \gamma_{0}\right)(1+$ $\left.2 \alpha \gamma_{0} t\right)$.
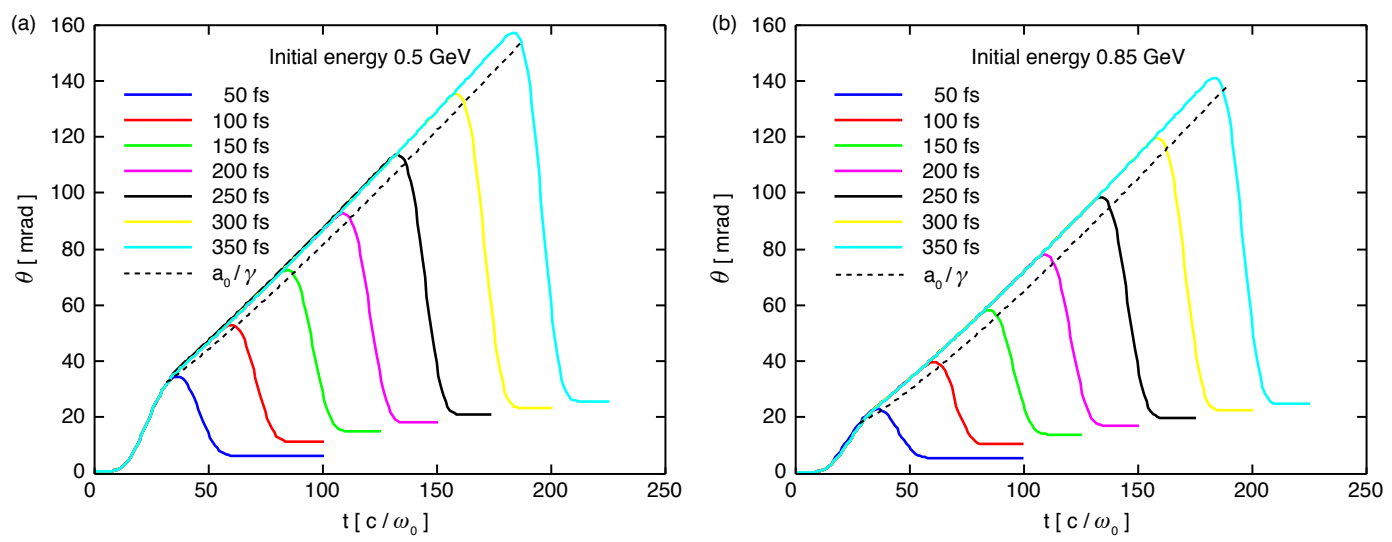

FIGURE 2. Electron beam divergence vs. time in the QED description. The electron beam initial energy is (a) $0.5 \mathrm{GeV}$ and (b) $0.85 \mathrm{GeV}$.

The dashed lines in Fig. 2 show the average expected angle $a_{0} / \gamma$ during the constant amplitude part of the laser envelope, where $\gamma$ is taken as the average relativistic factor of the electron bunch and $a_{0}=27$. We observe a similar trend with the simulation data, which indicates that the average divergence increase due to radiation emission in the constant amplitude region of the laser envelope is well explained by the semi-classical approach. However, there is a slight difference between the simulation data and the expected $a_{0} / \gamma$ which increases over time. After the interaction has finished, the electron beam has a residual divergence on the order of $\sim 10 \mathrm{mrad}$ which is larger than the initial divergence on the $0.2 \mathrm{mrad}$ level. With semi-classical radiation reaction models the final divergence would be very similar to the initial value (difference of aboout $0.01 \mathrm{mrad}$ ), which leaves us with the conclusion that the net beam divergence obtained in the laser interaction must be a consequence of the quantum stochasticity, even though the trend of average angle increase in a plane wave seems to be welldescribed by the semi-classical formula. To understand the origin of this effect, let us examine a transverse 
momentum phasespace in different instants of time shown in Fig. 3. In the beginning, the electron beam has a narrow momentum spread. Then, the laser interacts with the beam and introduces transversal momenta $p_{2}$ and $p_{3}$. During the plane wave stage the average $p_{\perp}$ indeed has an average value of $a_{0}$, but in the QED case there is a distribution around that value with a spread that is increasing with the interaction time. Finally, after the shutdown of the laser, there is a residual distribution in $p_{2}$ and $p_{3}$. The width of the final angular distribution increases slowly with the length of the total interaction time.
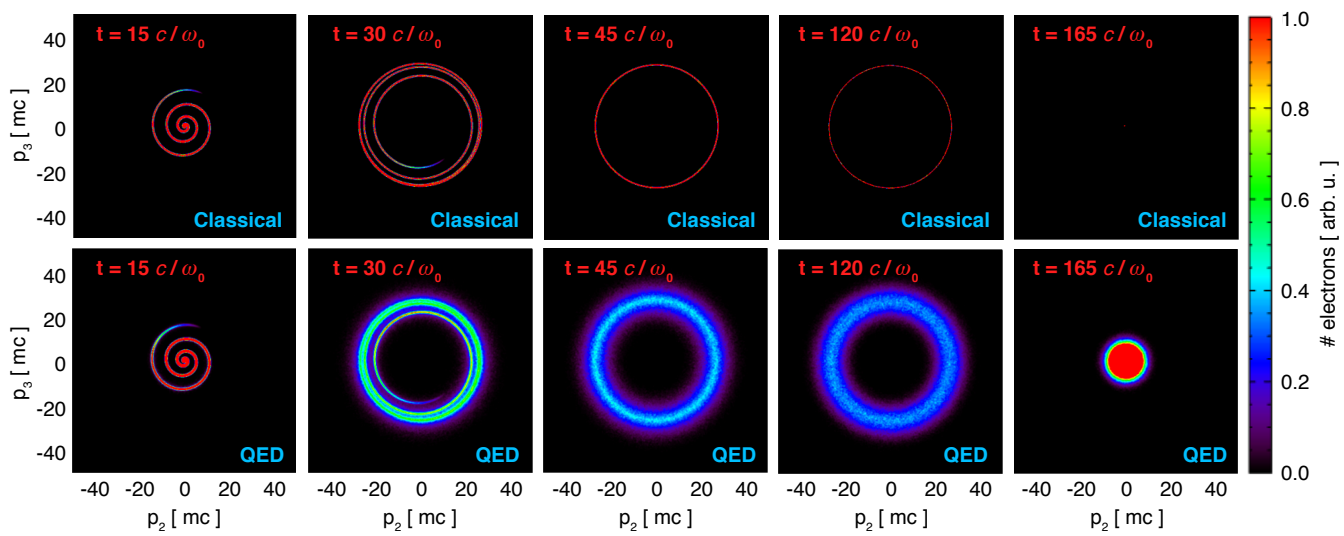

FIGURE 3. Transversal momentum space $p_{2}-p_{3}$ at different times. Classical and QED radiation reaction give different final transversal momentum spread.

\section{CONCLUSIONS}

In classical radiation reaction, the energy loss of one electron depends on its initial energy. For an electron beam, the main effects are the decrease in its mean energy and reduction of the energy distribution width. When QED effects are taken into account, the intrinsic stochastic nature of photon emission leads to diffusion in the energy distribution around the mean value. Therefore, in the quantum regime, there is a competition between these two tendencies. If we allow a long enough interaction time, there is a point when the diffusion is balanced by the energy width reduction. Beyond this point, the energy spread only decreases.

When including QED effects, the average divergence of the electron beam is well-described by the classical radiation reaction, however, when including the QED effects the distribution has a certain spread around this value that increases with the interaction time. This spread remains after the interaction is shut down and leads to a residual divergence of the electron beam.

\section{ACKNOWLEDGEMENTS}

This work is supported by the European Research Council (ERC-2010-AdG Grant 267841) and FCT (Portugal) grants PTDC/FIS/111720/2009, SFRH/BD/62137/2009 and SFRH/BD/39523/2007. Simulations were performed at Juqueen and Supermuc (Germany) under PRACE grants and IST cluster (Lisbon, Portugal).

\section{REFERENCES}

1. The Extreme Light Infrastructure, http://www.extreme-light-infrastructure.eu/.

2. N. V. Elkina et al., Phys. Rev. STAB 14, 054401 (2011).

3. C. P. Ridgers et al., Phys. Rev. Lett 108, 165006 (2012).

4. E. N. Nerush et al., Phys. Rev. Lett 106, 035001 (2011).

5. J. G. Kirk et al., PPCF 51, 085008 (2009).

6. A. Ilderton and G. Torgrimsson, Phys. Lett. B 725, 481 (2013).

7. N. Neitz and A. Di Piazza, Phys. Rev. Lett. 111, 054802 (2013).

8. T. G. Blackburn et al., Phys. Rev. Lett 112, 015001 (2014).

9. R. A. Fonseca et al., PPCF 55, 124001 (2013)

10. L. D. Landau and E. M. Lifshitz, The Classical Theory of Fields (Butterworth Heinemann, P, 1975).

11. T. Grismayer et al. in preparation

12. M. Vranic et al., arXiv:1306.0766 (2013).

13. D. M. Volkov, Z. Phys 94, 250-260 (1935).

14. A. I. Nikishov and V. I. Ritus, Sov. Phys JETP 25, 6 (1967). 